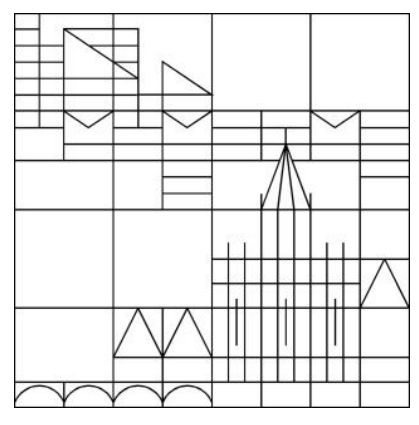

POD-based augmented Lagrangian method for state constrained heat-convection phenomena

\author{
Jonas Siegfried Jehle \\ Luca Mechelli \\ Stefan Volkwein
}

Konstanzer Schriften in Mathematik

Nr. 377, Juli 2018

ISSN 1430-3558

Konstanzer Online-Publikations-System (KOPS)

URL: http://nbn-resolving.de/urn:nbn:de:bsz:352-2-gh534968czve7

(C) Fachbereich Mathematik und Statistik

Universität Konstanz

Fach D 197, 78457 Konstanz, Germany 



\title{
POD-Based Augmented Lagrangian Method for State Constrained Heat-Convection Phenomena
}

Jonas Siegfried Jehle, Luca Mechelli, Stefan Volkwein

\begin{abstract}
We consider an optimal boundary control problem of the heat equation with convection with bilateral control and state constraints. The goal is to keep the temperature (state) within a desired range while the norm of the boundary controls is minimized. To numerically approximate the unique solution of the problem, a first-order augmented Lagrangian method is utilized. The gradient of the augmented Lagrangian is characterized by the solution of the adjoint equation. To decrease the computational time, we apply a reduced-order approach based on proper orthogonal decomposition (POD). To gain a sufficiently accurate approximation, we propose and compare different manners to compute the snapshots and thus the POD basis. Numerical tests compare the efficiency of the proposed strategies.
\end{abstract}

\section{Introduction}

Nowadays, the optimal control of parabolic partial differential equations is object of interest in many applications, e.g. in acoustics, electromagnetism and thermodynamics. In the literature, there are many contributions related to this field, like $[7,16]$. In particular, we are interested in the optimal control of convection-diffusion equation with bilateral control and state constraints, having energy efficient buildings in mind, i.e. the solution of the parabolic equation represents the temperature in a room and the heaters are seen as boundary controls; see $[14,15]$. To solve this problem, in contrast to [14], we use the augmented Lagrangian method, which can

Jonas Siegfried Jehle, Luca Mechelli, Stefan Volkwein

University of Konstanz, Department of Mathematics and Statistics, Universitätsstraße 10, D-78457

Konstanz, Germany, e-mail: Jonas.Jehle@uni-konstanz.de, Luca.Mechelli@uni-konstanz.de,

Stefan.Volkwein@uni-konstanz.de

Jonas Siegfried Jehle

Shanghai Jiao Tong University, Department of Mathematics, 800 Dongchuan Road, Shanghai 200240, China 
be seen as a penalization method that allows in our case to treat the state constraints by solving iteratively many only control-constrained optimal control problems; see $[3,4,9,10]$. This approach is different from the one in [15], where a virtual control concept is utilized (see [12]) that allows the use of a semi-smooth Newton method. For the numerical solution of the equations, we apply a Galerkin finite element approximation combined with an implicit Euler scheme in time and, in order to speedup the computation of optimal solutions, we build a reduced-order model based on POD; cf. [6, 8]. Since there is no computable a-priori POD error estimate, we compare different techniques. Moreover, being aware of methods to improve, e.g. TR-POD [1] and OS-POD [13], or to estimate, e.g. a-posteriori error estimator in [17], the quality of POD basis, we do not use them in this work.

The paper is organized in the following way: in Section 2 we introduce the optimal control problem; in Section 3 we describe how we adapt the augmented Lagrangian method to our setting and we briefly comment on the four methods we use to generate the snapshots to build the POD basis; in Section 4 numerical tests are shown and in Section 5 we draw some conclusions.

\section{The optimal control problem}

We consider an optimal control problem with time horizon $[0, T]$ with $T>0$. Let $\Omega \subset \mathbb{R}^{d}, d \in\{2,3\}$, be a bounded domain with Lipschitz-continuous boundary $\Gamma=\partial \Omega$. We suppose that $\partial \Omega$ is split into the two disjoint subsets $\Gamma_{\mathrm{c}}$ and $\Gamma_{\mathrm{o}}$, where at least $\Gamma_{\mathrm{c}}$ has nonzero (Lebesgue) measure. Let us set $Q=(0, T) \times \Omega$, $\Sigma_{\mathrm{c}}=(0, T) \times \Gamma_{\mathrm{c}}$, and $\Sigma_{\mathrm{o}}=(0, T) \times \Gamma_{\mathrm{o}}$. Moreover, let $H=L^{2}(\Omega), V=H^{1}(\Omega)$, $\mathcal{U}=L^{2}\left(0, T ; \mathbb{R}^{m}\right)$. We recall the Hilbert space

$$
W(0, T)=\left\{\varphi \in L^{2}(0, T ; V) \mid \varphi_{t} \in L^{2}\left(0, T ; V^{\prime}\right)\right\},
$$

where $V^{\prime}$ denotes the dual space of $V$; cf. [5]. We consider the following economic optimal control problem:

$$
\min J(x)=\frac{1}{2}\|u\|_{\mathfrak{U}}^{2}, \quad x=(y, u) \in \mathbb{X}:=W(0, T) \times \mathfrak{U}
$$

with $u \in \mathcal{U}$ boundary control for the state equation

$$
\begin{aligned}
y_{t}(t, x)-\Delta y(t, x)+v(t, x) \cdot \nabla y(t, x) & =0 & & \text { a.e. in } Q, \\
\frac{\partial y}{\partial n}(t, s)+y(t, s) & =\sum_{i=1}^{m} u_{i}(t) b_{i}(s) & & \text { a.e. on } \Sigma_{\mathrm{c}}, \\
\frac{\partial y}{\partial n}(t, s)+\gamma y(t, s) & =\gamma y_{\mathrm{out}}(t) & & \text { a.e. on } \Sigma_{\mathrm{o}}, \\
y(0, x) & =y_{\circ}(x) & & \text { a.e. in } \Omega,
\end{aligned}
$$


where $b_{1}, \ldots, b_{m} \in L^{\infty}\left(\Gamma_{\mathrm{c}}\right)$ are given control shape functions and 'a.e.' stands for 'almost everywhere'. Moreover, $u$ and $y$ have to satisfy the bilateral inequality constraints

$$
\begin{aligned}
u_{\mathrm{a} i}(t) & \leq u_{i}(t) \leq u_{\mathrm{b} i}(t), & & i=1, \ldots, m \text { a.e. in }[0, T], \\
y_{\mathrm{a}}(t, x) & \leq y(t, x) \leq y_{\mathrm{b}}(t, x) & & \text { a.e. in } Q .
\end{aligned}
$$

Assumption 1. In (1) we suppose that $\gamma \geq 0, y_{\text {out }} \in L^{2}(0, T), y_{\circ} \in H$, $v=\left(v_{1}, \ldots, v_{d}\right) \in L^{\infty}\left(0, T ; L^{\infty}\left(\Omega ; \mathbb{R}^{d}\right)\right), u_{\mathrm{a}}=\left(u_{\mathrm{a} i}\right)_{1 \leq i \leq m}, u_{\mathrm{b}}=\left(u_{\mathrm{b} i}\right)_{1 \leq i \leq m} \in \mathcal{U}$ and $y_{\mathrm{a}}, y_{\mathrm{b}} \in L^{2}(Q)$.

We introduce the time-dependent bilinear form $a(t ; \cdot, \cdot): V \times V \rightarrow \mathbb{R}$

$$
a(t ; \varphi, \phi)=\int_{\Omega} \nabla \phi \cdot \nabla \varphi+(v(t) \cdot \nabla \phi) \varphi \mathrm{d} x+\gamma \int_{\Gamma_{\mathrm{o}}} \varphi \phi \mathrm{d} s+\int_{\Gamma_{\mathrm{c}}} \varphi \phi \mathrm{d} s
$$

for $\varphi, \phi \in V$, the time-dependent linear functional $\mathscr{F}(t): V \rightarrow V^{\prime}$

$$
\langle\mathscr{F}(t), \varphi\rangle_{V^{\prime}, V}=\gamma y_{\text {out }}(t) \int_{\Gamma_{\mathrm{o}}} \varphi \mathrm{d} s \quad \text { for } \varphi \in V \text { a.e. in }[0, T],
$$

and the bounded linear operator $\mathscr{B}: \mathbb{R}^{m} \rightarrow V^{\prime}$ defined as

$$
\langle\mathscr{B} u, \varphi\rangle_{V^{\prime}, V}=\sum_{i=1}^{m} u_{i} \int_{\Gamma_{\mathrm{c}}} b_{i} \varphi \mathrm{d} s \quad \text { for all } \varphi \in V
$$

for given $u=\left(u_{i}\right) \in \mathbb{R}^{m}$. Now, a weak solution $y \in W(0, T)$ to (1b) for all $\varphi \in V$ satisfies the weak formulation

$$
\frac{d}{d t}\langle y(t), \varphi\rangle_{H}+a(t ; y(t), \varphi)=\langle\mathscr{F}(t)+\mathscr{B}(u(t)), \varphi\rangle_{V^{\prime}, V} \quad \text { a.e. in }(0, T]
$$

and $y(0)=y_{\circ}$ in $H$. By Assumption 1 the bilinear form $a(t ; \cdot, \cdot)$ is uniformly (w.r.t. the time $t$ ) continuous and weakly coercive, it is known that (2) admits a unique solution $y \in W(0, T)$; cf. [5]. Then we can define the bounded linear solution operator $\mathscr{S}: U \rightarrow W(0, T)$, such that $y=\mathscr{S} u$ is the unique weak solution of (1b) for $u \in U$. Furthermore, the set of admissible solutions is given by

$$
\mathbb{X}_{\mathrm{ad}}=\{x=(y, u) \in \mathbb{X} \mid x \text { satisfies (2), (1c) and (1d) }\} .
$$

Now, (1) can be expressed as

$$
\min \hat{J}(u):=J(\mathscr{S} u, u) \quad \text { subject to } \quad x=(y, u) \in \mathbb{X}_{\mathrm{ad}} .
$$

Note that $(\mathbf{P})$ is a linear-quadratic, strictly convex programming problem, so that there exists a unique optimal solution which is denoted by $\bar{x}=(\bar{y}, \bar{u})$. 


\section{Augmented Lagrangian Method}

In order to apply the augmented Lagrangian method to solve the optimal control problem $(\mathbf{P})$ we follow $[4,10]$. For $c \geq 0$ the augmented Lagrangian is given as

$$
\begin{aligned}
L_{c}\left(u, s_{\mathrm{a}}, s_{\mathrm{b}}, \mu_{\mathrm{a}}, \mu_{\mathrm{b}}\right)= & \hat{J}(u)+\left\langle\mu_{\mathrm{a}}, y_{\mathrm{a}}-\mathscr{S} u+s_{\mathrm{a}}\right\rangle_{L^{2}(Q)}+\left\langle\mu_{\mathrm{b}}, \mathscr{S} u-y_{\mathrm{b}}+s_{\mathrm{b}}\right\rangle_{L^{2}(Q)} \\
& +\frac{c}{2}\left\|y_{\mathrm{a}}-\mathscr{S} u+s_{\mathrm{a}}\right\|_{L^{2}(Q)}^{2}+\frac{c}{2}\left\|\mathscr{S} u-y_{\mathrm{b}}+s_{\mathrm{b}}\right\|_{L^{2}(Q)}^{2}
\end{aligned}
$$

where $s_{\mathrm{a}}, s_{\mathrm{b}}$ are non-negative slack variables used to transform the inequalities (1d) into equalities. From the first-order optimality conditions

$$
\begin{aligned}
& \frac{\partial L_{c}}{\partial s_{\mathrm{a}}}\left(u, s_{\mathrm{a}}, s_{\mathrm{b}}, \mu_{\mathrm{a}}, \mu_{\mathrm{b}}\right) \delta_{s_{\mathrm{a}}}=\left\langle\mu_{\mathrm{a}}, \delta_{s_{\mathrm{a}}}\right\rangle_{L^{2}(Q)}+c\left\langle y_{\mathrm{a}}-\mathscr{S} u+s_{\mathrm{a}}, \delta_{s_{\mathrm{a}}}\right\rangle_{L^{2}(Q)} \stackrel{!}{=} 0, \\
& \frac{\partial L_{c}}{\partial s_{\mathrm{b}}}\left(u, s_{\mathrm{a}}, s_{\mathrm{b}}, \mu_{\mathrm{a}}, \mu_{\mathrm{b}}\right) \delta_{s_{\mathrm{b}}}=\left\langle\mu_{\mathrm{b}}, \delta_{s_{\mathrm{b}}}\right\rangle_{L^{2}(Q)}+c\left\langle\mathscr{S} u-y_{\mathrm{b}}+s_{\mathrm{b}}, \delta_{s_{\mathrm{b}}}\right\rangle_{L^{2}(Q)} \stackrel{!}{=} 0
\end{aligned}
$$

we derive the two equalities

$$
\mu_{\mathrm{a}}+c\left(y_{\mathrm{a}}-\mathscr{S} u+s_{\mathrm{a}}\right)=0 \text { and } \mu_{\mathrm{b}}+c\left(\mathscr{S} u-y_{\mathrm{b}}+s_{\mathrm{b}}\right)=0 \quad \text { a.e. in } Q .
$$

Hence, to ensure $s_{\mathrm{a}} \geq 0$ and $s_{\mathrm{b}} \geq 0$, we set

$$
s_{\mathrm{a}}=\max \left\{0, \mathscr{S} u-y_{\mathrm{a}}-\frac{1}{c} \mu_{\mathrm{a}}\right\} \text { and } s_{\mathrm{b}}=\max \left\{0, y_{\mathrm{b}}-\mathscr{S} u-\frac{1}{c} \mu_{\mathrm{b}}\right\} .
$$

Then, as proved in [11], it holds

$$
\begin{aligned}
\hat{L}_{c}\left(u, \mu_{\mathrm{a}}, \mu_{\mathrm{b}}\right):=L_{c}\left(u, s_{\mathrm{a}}, s_{\mathrm{b}}, \mu_{\mathrm{a}}, \mu_{\mathrm{b}}\right)= & \hat{J}(u)-\frac{1}{2 c}\left(\left\|\mu_{\mathrm{a}}\right\|_{L^{2}(Q)}^{2}+\left\|\mu_{\mathrm{b}}\right\|_{L^{2}(Q)}^{2}\right) \\
& +\frac{c}{2}\left\|\max \left\{0, y_{\mathrm{a}}-\mathscr{S} u+\frac{\mu_{\mathrm{a}}}{c}\right\}\right\|_{L^{2}(Q)}^{2} \\
& +\frac{c}{2}\left\|\max \left\{0, \mathscr{S} u-y_{\mathrm{b}}+\frac{\mu_{\mathrm{b}}}{c}\right\}\right\|_{L^{2}(Q)}^{2} .
\end{aligned}
$$

Therefore, to find the optimal solution of $(\mathbf{P})$, we can use Algorithm 1.

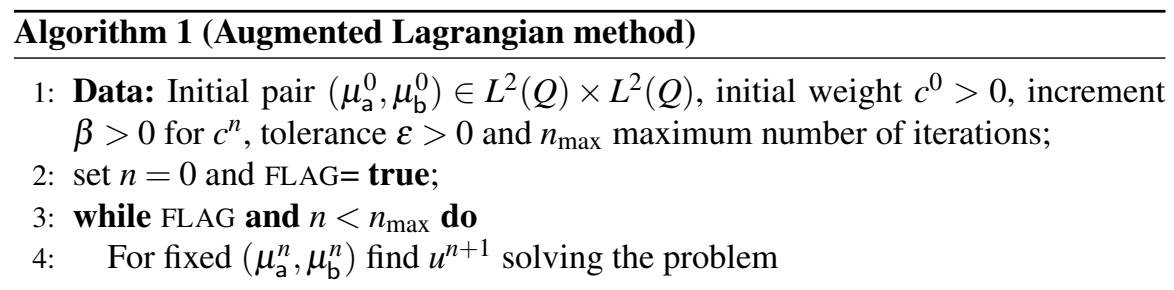




$$
\begin{aligned}
\min \hat{L}_{c^{n}}\left(u, \mu_{\mathrm{a}}^{n}, \mu_{\mathrm{b}}^{n}\right)= & \hat{J}(u)+\frac{c^{n}}{2}\left\|\max \left\{0, y_{\mathrm{a}}-\mathscr{S} u+\frac{\mu_{\mathrm{a}}^{n}}{c^{n}}\right\}\right\|_{L^{2}(Q)}^{2} \\
& +\frac{c^{n}}{2}\left\|\max \left\{0, \mathscr{S} u-y_{\mathrm{b}}+\frac{\mu_{\mathrm{b}}^{n}}{c^{n}}\right\}\right\|_{L^{2}(Q)}^{2} \\
& -\underbrace{\frac{1}{2 c^{n}}\left(\left\|\mu_{\mathrm{a}}^{n}\right\|_{L^{2}(Q)}^{2}+\left\|\mu_{\mathrm{b}}^{n}\right\|_{L^{2}(Q)}^{2}\right)}_{\text {independent of } u} \\
\text { s.t. } u & \in \mathcal{U}_{\mathrm{ad}}:=\{u \in \mathcal{U} \mid u \text { satisfies (1c) }\},
\end{aligned}
$$

5: Update the Lagrange multipliers

$$
\begin{aligned}
& \mu_{\mathrm{a}}^{n+1}=\max \left\{0, \mu_{\mathrm{a}}^{n}+c^{n}\left(y_{\mathrm{a}}-\mathscr{S} u^{n+1}\right)\right\}, \\
& \mu_{\mathrm{b}}^{n+1}=\max \left\{0, \mu_{\mathrm{b}}^{n}+c^{n}\left(\mathscr{S} u^{n+1}-y_{\mathrm{b}}\right)\right\} ;
\end{aligned}
$$

6: $\quad$ if $\left\|u^{n}-u^{n+1}\right\| u \leq \varepsilon$ then

7: $\quad$ FLAG $=$ false

8: $\quad$ end if

9: $\quad$ set $c^{n+1}=\beta c^{n}$ and $n=n+1$;

10: end while

Remark 1. 1) To solve the minimization problem $\left(\mathbf{L}^{n}\right)$, we use the projected gradient method (due to the control constraints). In future works, we want to utilize an inexact version of Algorithm 1 within a Model Predictive Control approach, as done for the primal dual active set strategy in [15]. The idea is then to compare the two different approaches in terms of approximation of the feedback control $u$ and of the computational time, for more details we refer to [11].

2) To apply the projected gradient algorithm, we need the partial derivative with respect to $u$ of the reduced Lagrangian $\hat{L}_{c}\left(u, \mu_{\mathrm{a}}, \mu_{\mathrm{b}}\right)$, i.e.:

$$
\frac{\partial \hat{L}_{c}}{\partial u}\left(u, \mu_{\mathrm{a}}, \mu_{\mathrm{b}}\right) u^{\delta}=\left\langle-\mathscr{B}^{\star} p+u, u^{\delta}\right\rangle_{\mathfrak{u}} \quad \text { for } u^{\delta} \in \mathcal{U}
$$

with the solution $p$ of the adjoint equation

$$
-\frac{d}{d t}\langle p(t), \varphi\rangle_{H}+a(t ; \varphi, p(t))=\left\langle\mathscr{G}\left(t ; y(t), \mu_{\mathrm{a}}(t), \mu_{\mathrm{b}}(t)\right), \varphi\right\rangle_{V^{\prime}, V}
$$

for all $\varphi \in V$ a.e. in $[0, T]$ and $p(T)=0$ in $H$. Further, $\mathscr{G}(t ; \cdot, \cdot, \cdot): V \times L^{2}(Q) \times L^{2}(Q) \rightarrow V^{\prime}$ is defined as

$$
\begin{aligned}
\langle\mathscr{G}(t ; \theta, \phi, \psi), \varphi\rangle_{V^{\prime}, V}= & c \int_{\Omega} \max \left\{0, y_{\mathrm{a}}(t)-\theta+\frac{\phi}{c}\right\} \varphi \mathrm{d} x \\
& -c \int_{\Omega} \max \left\{0, \theta-y_{\mathrm{b}}(t)+\frac{\psi}{c}\right\} \varphi \mathrm{d} x .
\end{aligned}
$$


3) For implementing Algorithm 1 numerically, we discretize (2) and (4): for the temporal discretization we utilize the implicit Euler method, while the spatial variable is approximated utilizing a reduced-order Galerkin approach; cf. [2, 14]. Here, we apply the POD method, where the snapshots are computed from piecewise linear finite element (FE) solutions of the state and dual variables; cf. [6, 8]. In Section 4, we compare three different methods to compute the POD basis:

- (POD-M1): Use the optimal control $\bar{u}$ to compute state and adjoint snapshots.

- (POD-M2): Use a random control $\widetilde{u} \in \mathcal{U}_{\text {ad }}$ to compute state and adjoint snapshots.

- (POD-M3): Use a sub-optimal control $\hat{u}$, generated utilizing FE discretization for $n=1,2,3$ in Algorithm 1, to compute state and adjoint snapshots.

4) Due to the different magnitudes of state and adjoint snapshots, we observed that it is convenient to scale them by two different values respectively, in order to balance the optimization problem involved in the basis computation. This procedure does not change the set spanned by the snapshots, but improves the POD basis approximation, as shown in Section 4. After several tests with different scaling values (e.g. $L^{2}$ norms, mean values), we choose to report the results for the best choice we have found: we divide each state snapshot by variance $\sigma^{2}$ defined as

$$
\sigma^{2}=\frac{1}{N t} \sum_{i=0}^{N t-1}\left(\left\|y\left(t_{i}\right)\right\|_{H}-\mu\right)^{2}, \text { with } \mu=\frac{1}{N t} \sum_{i=0}^{N t-1}\left\|y\left(t_{i}\right)\right\|_{H}
$$

and we do similarly for the adjoint ones. When this approach is utilized, we report each method with the name varPOD from now on.

\section{Numerical Tests}

The tests in this section are implemented on a Notebook Acer Aspire E15 E5-71G-717X, Intel ${ }^{\circledR}$ Core $^{\mathrm{TM}}$ i7-5500U $2.4 \mathrm{GHz}$ (with Turbo Boost up to $3.0 \mathrm{GHz}$ ) and 8GB RAM in the programming language MATLAB. For the tests, we set $T=1$ and the unit square $\Omega=(0,1) \times(0,1)$ represents our room. The controls, i.e. the heaters, are placed as shown in Fig. 1 with related shape functions:

$$
b_{1}(x)=\left\{\begin{array}{l}
1 \text { for } x_{1}=0,0 \leq x_{2} \leq 0.25, \\
0 \text { otherwise }
\end{array} \quad b_{2}(x)=\left\{\begin{array}{l}
1 \text { for } x_{1}=1,0.75 \leq x_{2} \leq 1 \\
0 \text { otherwise }
\end{array}\right.\right.
$$

respectively. For the state equation, we choose the physical parameter $\gamma=0.05$, the initial condition $y_{\circ}(x)=x_{1}^{2}+x_{2}^{2}$ for $x=\left(x_{1}, x_{2}\right) \in \Omega$ and the outside temperature $y_{\text {out }}(t)=2 t-1$ for all $t \in[0, T]$. 


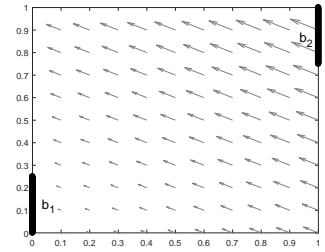

(a) $t<0.5$

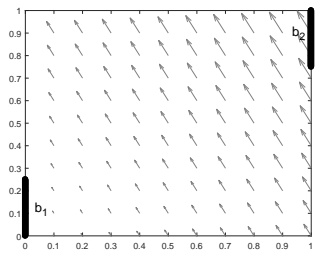

(b) $t \geq 0.5$

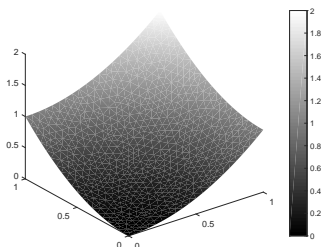

Fig. 1 Spatial domain $\Omega$ with the two boundary controls and the velocity fields (grey arrows) at two time istances (left and middle); initial condition $y_{\circ}(x)$ (right).

The components of the velocity field $v(t, x)$ are respectively

$$
v_{1}(t, x)=\left\{\begin{array}{ll}
-x_{1}-x_{2} \text { for } 0 \leq t<0.5, \\
\frac{-x_{1}-x_{2}}{2} & \text { for } 0.5 \leq t \leq T,
\end{array} v_{2}(t, x)= \begin{cases}\frac{x_{1}+x_{2}}{2} \text { for } 0 \leq t<0.5, \\
x_{1}+x_{2} \text { for } 0.5 \leq t \leq T,\end{cases}\right.
$$

for $t \in[0, T], x \in \Omega$. Furthermore, we take $y_{\mathrm{a}}(t)=3 t, y_{\mathrm{b}}(t)=4$ for $t \in[0, T]$ as the state bounds and $u_{\mathrm{a} i}(t)=0, u_{\mathrm{b} i}(t)=12$ for $t \in[0, T], i=1, \ldots, m$ as the control bounds. For the implicit Euler method, we use the equidistant time step $\Delta t=0.01$. The spatial discretization is built by piecewise linear FE on a triangular mesh with $N_{x}=712$ nodes. As initial guess for the control, we choose $u_{0}(t)=0$ for $t \in[0, T]$. In Table 1, for different values of $c^{0}$ and $\beta$ and different spatial discretization, we report the number of iteration needed by the projected gradient method to converge in step 4 of Algorithm 1 for any iteration $n$. Additionally, the value of the cost functional $\hat{J}(\bar{u})$, the number of active points for the state bounds $|\mathscr{A}|$ and the computational time speed-up due to the POD approximation are shown in Table 2 together with

$$
\left|\mathscr{A}^{\mathrm{FE}} \Delta \mathscr{A}^{\mathrm{POD}}\right|=\left|\mathscr{A}^{\mathrm{FE}} \cup \mathscr{A}^{\mathrm{POD}}-\mathscr{A}^{\mathrm{FE}} \cap \mathscr{A}^{\mathrm{POD}}\right|
$$

as well as the POD relative error for the controls defined as

$$
\text { rel.err. } u=\frac{\left\|u^{\mathrm{FE}}-u^{\mathrm{POD}}\right\|_{u}}{\left\|u^{\mathrm{FE}}\right\|_{\mathfrak{u}}}
$$

From these tables, one can observe that with both choices of $c^{0}$ and $\beta$ the optimal control problem reaches the unique optimal solution as expected. The number of active points for the optimal state is significantly small compared to the total number of space-time grid points: $N_{x} \times N_{t}=712 \times 101=71912$ and it can be even reduced setting different tolerances for the augmented Lagrangian $(\varepsilon)$ and the project gradient $\left(\varepsilon_{g p}\right)$ methods. In Fig. 2, we can notice that the optimal solution, in particular the average temperature, is almost everywhere inside the bounds and, when it is not, there are only small differences, which are related to the numerical tolerances chosen. In Table 2, it is shown also that the quality of the POD approximation is improved after rescaling the snapshots with their variance $\sigma^{2}$, in particular POD-M1 results can be significantly improved. As already mentioned, when the snapshots of POD-M1 are 
Table 1 Results for different combinations of $c^{0}$ and $\beta$ (augmented Lagrangian tolerance $\varepsilon=$ $10^{-5}$, projected gradient tolerance $\varepsilon_{g p}=0.2 \varepsilon$, number of POD basis $\ell=18, n_{\max }=150$ ).

\begin{tabular}{lllll}
\hline Discretiz. & $c^{0}$ & $\beta$ & Projected gradient iterations & $n$ \\
\hline FE & 1 & 4 & $\{4,4,4,286,3,141,155,131,308,252,279,198,2\}$ & 13 \\
POD-M1 & 1 & 4 & $\{4,4,4,298,3,160,167,153,476,7,2\}$ & 11 \\
varPOD-M1 & 1 & 4 & $\{4,4,4,286,3,141,155,131,308,252,279,198,2\}$ & 13 \\
POD-M2 & 1 & 4 & $\{4,4,4,286,3,141,154,125,304,255,1000,4,2\}$ & 13 \\
varPOD-M2 & 1 & 4 & $\{4,4,4,286,3,141,155,131,309,250,280,202,2\}$ & 13 \\
POD-M3 & 1 & 4 & $\{4,4,4,286,3,142,154,111,374,434,126,2\}$ & 12 \\
varPOD-M3 & 1 & 4 & $\{4,4,4,286,3,141,155,131,308,250,275,200,2\}$ & 13 \\
FE & 0.1 & 5 & $\{4,4,119,4,677,3,209,134,297,249,309,211,2\}$ & 13 \\
POD-M1 & 0.1 & 5 & $\{4,4,120,4,680,3,216,139,234,316,602,184,2\}$ & 13 \\
varPOD-M1 & 0.1 & 5 & $\{4,4,119,4,677,3,209,134,297,249,309,210,2\}$ & 13 \\
POD-M2 & 0.1 & 5 & $\{4,4,119,4,677,3,209,134,299,249,312,166,2\}$ & 13 \\
varPOD-M2 & 0.1 & 5 & $\{4,4,119,4,677,3,209,134,296,249,309,212,2\}$ & 13 \\
POD-M3 & 0.1 & 5 & $\{4,4,119,4,677,3,208,131,106,160,274,6,2\}$ & 13 \\
varPOD-M3 & 0.1 & 5 & $\{4,4,119,4,677,3,209,134,300,249,310,209,2\}$ & 13 \\
\hline
\end{tabular}

not scaled they have huge differences in magnitude, due to the fact that the adjoint variable magnitude at each time step depends directly on $c^{13}=4^{12} \simeq 1.7 \times 10^{7}$ (or $\left.c^{13}=0.1 \times 5^{12} \simeq 2.4 \times 10^{7}\right)$. Further, this improved approximation is also appearing for POD-M2 and POD-M3, even if there is less effectiveness compared to POD-M1 because the value of $c$ is way smaller than $10^{7}$. The speed-up is around 15 for all the methods, except for POD-M3 and varPOD-M3 in case of $c^{0}=0.1$ and $\beta=5$ where it is even lower than 10 . This can be justified from the fact that when we compute the FE suboptimal control $\hat{u}$ for $n=3$, the projected gradient method takes 119 iterations to converge, so the idea of fixing a maximum number of finite element it-

Table 2 Cost functional $\hat{J}(\bar{u})$, state active points, POD errors and computational time speed-up.

\begin{tabular}{llllrcrc}
\hline Discretiz. & $c^{0}$ & $\beta$ & $\hat{J}(\bar{u})$ & $|\mathscr{A}|$ & rel.err. $u$ & $\left|\mathscr{A}^{\mathrm{FE}} \Delta \mathscr{A}^{\mathrm{POD}}\right|$ & Speed-up \\
\hline FE & 1 & 4 & 82.12 & 94 & - & - & - \\
POD-M1 & 1 & 4 & 91.89 & 590 & $7.78 \times 10^{-2}$ & 548 & 20.7 \\
varPOD-M1 & 1 & 4 & 82.12 & 95 & $5.02 \times 10^{-4}$ & 1 & 15.2 \\
POD-M2 & 1 & 4 & 82.06 & 101 & $1.58 \times 10^{-2}$ & 57 & 11.7 \\
varPOD-M2 & 1 & 4 & 82.17 & 91 & $1.95 \times 10^{-3}$ & 3 & 15.1 \\
POD-M3 & 1 & 4 & 79.88 & 175 & $4.77 \times 10^{-2}$ & 167 & 14.8 \\
varPOD-M3 & 1 & 4 & 82.14 & 93 & $4.92 \times 10^{-3}$ & 1 & 13.8 \\
FE & 0.1 & 5 & 82.07 & 95 & - & - & - \\
POD-M1 & 0.1 & 5 & 95.51 & 299 & $1.98 \times 10^{-1}$ & 270 & 13.4 \\
varPOD-M1 & 0.1 & 5 & 82.08 & 95 & $5.31 \times 10^{-4}$ & 0 & 14.9 \\
POD-M2 & 0.1 & 5 & 82.04 & 98 & $5.06 \times 10^{-3}$ & 3 & 14.6 \\
varPOD-M2 & 0.1 & 5 & 82.12 & 93 & $3.20 \times 10^{-3}$ & 2 & 15.8 \\
POD-M3 & 0.1 & 5 & 83.68 & 343 & $8.48 \times 10^{-2}$ & 304 & 9.6 \\
varPOD-M3 & 0.1 & 5 & 82.04 & 96 & $3.42 \times 10^{-3}$ & 3 & 8.2 \\
\hline
\end{tabular}


erations should be considered to generate the suboptimal control $\hat{u}$ for improving the computational time speed-up, of course paying in accuracy of the approximation.

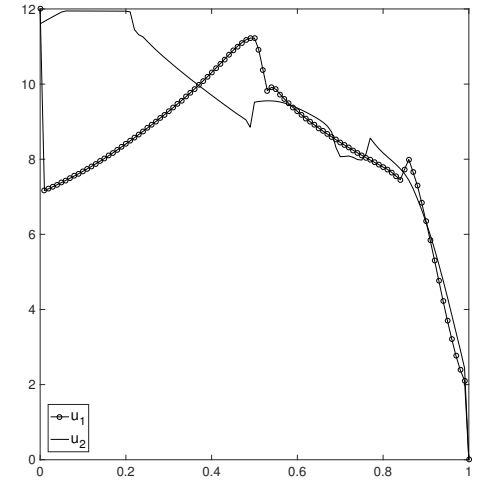

(a) Optimal controls for each time instance.

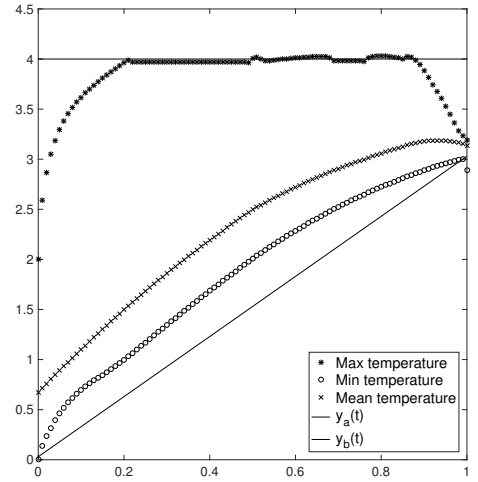

(b) Temperature for each time instance.

Fig. 2 Plots of optimal solution computed with FE with $c^{0}=1$ and $\beta=4$.

\section{Conclusion}

To conclude, in contrast to [14], we have applied a first-order augmented Lagrangian method to solve the optimal control problem with bilateral control and state constraints. Except for few points (compared to the total number of nodes in the spacetime grid), we got the possibility to stay inside the state bounds as well. Therefore, it is meaningful to proceed by combining this method with a model predictive control approach, following the idea of [15]. Moreover, to speed-up the computational process, we have combined the previous algorithm with POD and we have compared different approaches to generate the snapshots. We have also improved the quality of the POD approximation scaling state and adjoint snapshots by different values, to balance the optimization process better which is involved in the POD basis' computation. This technique turned out to be successful: for the same number of basis $\ell$ we have that the relative errors of varPOD methods are lower than the POD relative ones without affecting the computational time speed-up.

Acknowledgements Luca Mechelli gratefully acknowledges support by the German Science Fund DFG grant Reduced-Order Methods for Nonlinear Model Predictive Control. 


\section{References}

[1] Arian, E.; Fahl M.; Sachs, E.W.: Trust-region proper orthogonal decomposition for flow control. Technical Report 2000-25, ICASE (2000).

[2] Benner, P.; Cohen, A.; Ohlberger, M.; Willcox, K.: Model Reduction and Approximation: Theory and Algorithms. Philadelphia: SIAM, 2017.

[3] Bergounioux, M.: Augmented Lagrangian method for distributed optimal control problems with state constraints. Journal of Optimization Theory and Applications, Vol. 78, No. 3, pp. 493521, 1993.

[4] Bertsekas, D.P.: Constrained Optimization and Lagrange Multiplier Methods. Belmont: Athena Scientific, 1996.

[5] Dautray, R.; Lions, J.L.: Mathematical Analysis and Numerical Methods for Science and Technology. Volume 5: Evolution Problems I. Berlin: Springer-Verlag, 2000.

[6] Gubisch, M.; Volkwein, S.: Proper orthogonal decomposition for linear-quadratic optimal control. Chapter 1 in [2].

[7] Hinze, M. ; Pinnau, R.; Ulbrich, M.; Ulbrich, S.: Optimization with PDE Constraints. Berlin: Springer-Verlag, 2009.

[8] Holmes, P.; Lumley, J.L; Berkooz, G.; Rowley, C.W.: Turbulence, Coherent Structures, Dynamical Systems and Symmetry, 2nd ed. Cambridge: Cambridge Monographs on Mechanics. Cambridge University Press, 2012.

[9] Ito, K.; Kunisch, K.: Augmented Lagrangian methods for nonsmooth, convex optimization in Hilbert spaces. Nonlinear Analysis: Theory, Methods and Applications, Vol. 41, pp. 591-616, 2000.

[10] Ito, K.; Kunisch, K.: Lagrange Multiplier Approach to Variational Problems and Applications. Philadelphia: Advances in Design and Control, SIAM, 2008.

[11] Jehle, J.S.: First-order augmented Lagrangian methods for state constraint problems. Master's thesis. To appear in KOPS - The Institutional Repository of the University of Konstanz, 2018.

[12] Krumbiegel, K.; Rösch, A.: A virtual control concept for state constrained optimal control problems. Computational Optimization and Applications, Vol. 43, pp. 213-233, 2009.

[13] Kunisch, K.; Volkwein, S.: Proper orthogonal decomposition for optimality systems. ESAIM: M2AN, Vol. 42, pp. 1-23, 2008.

[14] Mechelli, L.; Volkwein, S.: POD-based economic optimal control of heatconvection phenomena. Submitted (2017), preprint download at: http://nbnresolving.de/urn:nbn:de:bsz:352-2-au6ei3apyzpv0

[15] Mechelli, L.; Volkwein, S.: POD-based economic model predictive control for heat-convection phenomena. Submitted (2017), preprint download at: http://nbnresolving.de/urn:nbn:de:bsz:352-2-17c2ahte39kao3

[16] Tröltzsch, F.: Optimal Control of Partial Differential Equations. Theory, Methods and Applications. Providence: American Mathematical Society, 2010.

[17] Tröltzsch, F.; Volkwein, S.: POD a-posteriori error estimates for linear-quadratic optimal control problems. Computational Optimization and Applications, Vol. 44, pp. 83-115, 2009. 